\section{Crisper results for CRISPR}

\section{By Kai-Jye Lou, Senior Writer}

Since its introduction earlier this year, proponents of CRISPR-based genome editing have touted its simplicity and efficiency over other genome editing platforms, but they have more recently also offered a word of caution on its specificity. ${ }^{1-3}$ Research from the Broad Institute of MIT and Harvard may now allay these concerns with an updated version of the technique that decreases editing activity at predicted offtarget sites by 50 - to 1,000-fold compared with earlier implementations. ${ }^{4}$

The group now needs to assess the approach's off-target editing activity on the whole-genome level.

CRISPR (clustered, regularly interspaced short palindromic repeats)based genome editing, and more specifically CRISPR-Cas9 (CRISPRassociated protein 9)-based genome editing, came into the limelight this January after multiple research groups showed proof of concept for engineering site-specific mutations in the genomes of key model organisms including bacterial and mammalian cells, zebrafish and rodents..$^{5-11}$

Less than four months after this initial flurry of results, one of the groups published the use of the CRISPR-Cas9 system to simultaneously engineer point mutations into multiple target genes in a mouse, ${ }^{12,13}$ which typically is a time- and labor-intensive process that usually would require several rounds of interbreeding.

The platform is derived from an acquired immunity-like system in bacteria, whereby short CRISPR-encoded RNAs guide CRISPRassociated proteins such as the Cas9 endonuclease to cleave homologous foreign DNA contained within plasmids or bacteriophages.

In the CRISPR-Cas9 platform, the specificity of the Cas9 endonuclease is dictated by base pairing between a custom-designed single guide RNA (sgRNA) and the target DNA sequence on the host genome. This property greatly increases the system's ease of use compared with zinc finger nuclease (ZFN)- and transcription activatorlike effector nuclease (TALEN)-based genome editing.

Those platforms use custom-designed DNA-binding protein domains or amino acid repeats to guide site-specific DNA editing. Moreover, the ZFN- and TALEN-based platforms generally are not amenable to the simultaneous introduction of mutations in multiple target genes, which makes such methods less efficient than CRISPR-Cas9 platforms.

However, a trio of studies published in Nature Biotechnology over the past three months by some of the same research groups involved in the development of the system has shown that CRISPR-Cas9 genome editing could have a high rate of off-target mutagenesis because the Cas 9 endonuclease can cleave DNA sequences that have a few mismatched nucleotides with the guide RNA.

This specificity issue could make it difficult for researchers to definitively link a gene to a particular phenotype when using CRISPRCas9 genome editing to create new model organisms.

To address the issue, a team at the Broad Institute led by Feng Zhang took a lesson from ZFN and TALEN systems, which use two independent DNA-binding modules to control specificity. The group developed an analogous approach for the CRISPR-Cas9 system that uses specifically paired guide RNAs that bind to opposite DNA strands at a target locus and a mutant nickase version of Cas9.

Whereas wild-type Cas9 has two nuclease domains that allow it to create double-stranded breaks when an sgRNA binds to a target DNA sequence, the Cas9 nickase used by Zhang's group has only one active nuclease domain; thus, two guide RNAs are required to bind at specific offset sites on opposite DNA strands to engage a Cas9 nickase on each strand to create the double-stranded break-a so-called double-nicking strategy.

A group at Seoul National University reported a similar doublenicking approach last year that uses zinc finger nickases as opposed to nucleases to improve the specificity of genome editing. ${ }^{14}$

"In developing the double-nicking system, we thought that, analogous to the requirement of dimerization for ZFNs and TALENs, if we could require two independently targeted nicking events to induce a double-stranded break, we could significantly decrease the off-target activity of the CRISPR system," said Zhang, a core member at the Broad Institute.

In cell lines, mutant Cas9 plus paired guide RNAs decreased cleavage at predicted off-target cleavage sites by 50 - to 1,000 -fold compared with wild-type Cas 9 plus sgRNAs. Importantly, this decrease in off-target cleavage activity did not come at the expense of on-target cleavage efficiency.

The measured on-target cleavage efficiency of the mutant Cas9 was at least $80 \%$ and similar to that of wild-type Cas9. Off-target activity of the mutant Cas9 can still create nicks in one of the two DNA strands, but these are rapidly repaired by the host cell.

Results were published in Cell.

"The efficiency and the ease of use of the Cas9sgRNA system has allowed rapid and exciting work across a number of cell types and organisms already this
"It is clear that at least for some implementations of the technology, improvements on specificity will be nicking strategy is an exciting example of such an improvement."

-Rachel Haurwitz, Caribou Biosciences Inc. necessary, and the doubleyear, but questions around specificity have lingered," said Rachel Haurwitz, cofounder, president and CEO of Caribou Biosciences Inc. "It is clear that at least for some implementations of the technology, improvements on specificity will be necessary, and the double-nicking strategy is an exciting example of such an improvement." 
Jennifer Doudna, a cofounder of Caribou, added, "The use of a nickase form of Cas9 to generate two closely spaced nicks in doublestranded DNA enhances targeting specificity of this system to a level that will be potentially suitable for applications such as human therapeutics." Doudna is a professor of biochemistry, biophysics and structural biology at the University of California, Berkeley and a Howard Hughes Medical Institute investigator.

Haurwitz cautioned, however, that a possible trade-off with the double-nicking strategy could be a reduction in the number of genomic sites that can be targeted because it now requires two target sites in opposite orientation that are within a specific range of one another.

Caribou is developing and commercializing its own implementations of the CRISPR-Cas9 genome editing system. Haurwitz declined to disclose details.

\section{Getting the whole-genome picture}

The current study only looked for activity at loci predicted to be offtarget modification sites based on sequence similarity with the target DNA. Thus, the next step is to better characterize the off-target activity rate of the updated approach at the genomewide level-especially at nonpredicted modification sites.

Other aspects of the CRISPR-Cas9 platforms that should be prioritized for refinement include the delivery vehicles currently used to get the system's components into cells, said Jin-Soo Kim, an associate professor at Seoul National University and cofounder of ToolGen Inc.

He noted that the current standard delivery approach, plasmid transfection, can be difficult to do in some cell types, such as human stem cells.

ToolGen offers custom CRISPR-based services to researchers.
Doudna added that a better understanding of how to design the guide RNAs is another area that warrants further exploration.

The Broad Institute has filed a patent application covering the work reported in Cell. The technology is available for licensing.

Lou, K.-J. SciBX 6(35); doi:10.1038/scibx.2013.950

Published online Sept. 12, 2013

\section{REFERENCES}

1. Fu, Y. et al. Nat. Biotechnol. 31, 822-826 (2013)

2. Hsu, P.D. et al. Nat. Biotechnol. 31, 827-832 (2013)

3. Mali, P. et al. Nat. Biotechnol. 31, 833-838 (2013)

4. Ran, F.A. et al. Cell; published online Aug. 29, 2013; doi:10.1016/j.cell.2013.08.021

Contact: Feng Zhang, Broad Institute of MIT and Harvard, Cambridge, Mass. e-mail: zhang@broadinstitute.org

5. Cong, L. et al. Science 339, 819-823 (2013)

6. Mali, P. et al. Science 339, 823-826 (2013)

7. Hwang, W.Y. et al. Nat. Biotechnol. 31, 227-229 (2013)

8. Jinek, M. et al. eLife 2, e00471; published online Jan. 29, 2013; doi:10.7554/eLife.00471

9. Cho, S.W. et al. Nat. Biotechnol. 31, 230-232 (2013)

10. Jiang, W. et al. Nat. Biotechnol. 31, 233-239 (2013)

11. Cain, C. SciBX 6(4); doi:10.1038/scibx.2013.77

12. Wang, H. et al. Cell 153, 910-918 (2013)

13. Cain, C. SciBX 6(19); doi:10.1038/scibx.2013.455

14. Kim, E. et al. Genome Res. 22, 1327-1333 (2012)

COMPANIES AND INSTITUTIONS MENTIONED

Broad Institute of MIT and Harvard, Cambridge, Mass. Caribou Biosciences Inc., Berkeley, Calif. Howard Hughes Medical Institute, Chevy Chase, Md. Seoul National University, Seoul, South Korea ToolGen Inc., Seoul, South Korea University of California, Berkeley, Calif. 